\title{
Pauli blocking and medium effects in nucleon knockout reactions
}

\author{
C. A. Bertulani ${ }^{1, *}$ and C. De Conti ${ }^{2, \dagger}$ \\ ${ }^{1}$ Physics Department, Texas A\&M University-Commerce, Commerce, Texas 75428-3011, USA \\ ${ }^{2}$ Campus Experimental de Itapeva, Universidade Estadual Paulista, 18409-010 Itapeva, SP, Brazil
}

(Received 12 April 2010; published 3 June 2010)

\begin{abstract}
We study medium modifications of the nucleon-nucleon $(N N)$ cross sections and their influence on the nucleon knockout reactions. Using the eikonal approximation, we compare the results obtained with free $N N$ cross sections with those obtained with a purely geometrical treatment of Pauli blocking and with $N N$ obtained with more elaborated Dirac-Bruecker methods. The medium effects are parametrized in terms of the baryon density. We focus on symmetric nuclear matter, although the geometrical Pauli blocking also allows for the treatment of asymmetric nuclear matter. It is shown that medium effects can change the nucleon knockout cross sections and momentum distributions up to $10 \%$ in the energy range $E_{\text {lab }}=50-300 \mathrm{MeV} /$ nucleon. The effect is more evident in reactions involving halo nuclei.
\end{abstract}

DOI: 10.1103/PhysRevC.81.064603

PACS number(s): 25.60.Gc, 21.65.-f

\section{INTRODUCTION}

Nuclear structure calculations are now able to reproduce the measured masses, charge radii, and low-lying excited states of a large number of nuclei. For very exotic nuclei, the small additional stability that comes with the filling of a particular orbital can have profound effects upon their existence as bound systems, their lifetime, and structure. The determination of the ordering, spacing, and the occupancy of orbitals is therefore essential in assessing how exotic nuclei evolve in the presence of large neutron or proton excess and to what extent theories have predictive power. Such spectroscopy of the single-particle structure in short-lived nuclei typically uses direct nuclear reactions.

Nucleon knockout reactions at intermediate energies have become a well-established and quantitative tool for studying the location and occupancy of single-particle states and correlation effects in the nuclear many-body system, as discussed in Refs. [1-5]. In a peripheral, sudden collision of the fast-moving projectile, a single nucleon is removed from the projectile, producing projectilelike residues in the exit channel [4]. Referred to the center-of-mass system of the projectile, the transferred momentum is $\mathbf{k}_{c}$. In the sudden approximation and for the knockout reaction, this must equal the momentum of the struck nucleon before the collision. The measured partial cross-sections to individual final levels provide spectroscopic factors for the individual angular-momentum components $j$. In complete analogy to the use of angular distributions in transfer reactions, the orbital angular momentum $l$ is in the knockout reactions revealed by the distributions of the quantity $\mathbf{k}_{c}$.

Extensions of the nucleon knockout formalism including the treatment of final-state interactions were discussed in Ref. [6], where it is shown that Coulomb final-state interactions are of relevance. They can be done by just adding the Coulomb phase $\phi=\phi_{N}+\phi_{C}$ in the eikonal phase, as

\footnotetext{
*carlos_bertulani@tamu-commerce.edu

†conti@itapeva.unesp.br
}

described [6]. Inclusion of higer-order effects [7,8] and a theory for two-nucleon knockout [9-11] has been developed. Knockout reactions represent a particular case for which higher projectile energies allow a simpler theoretical treatment of the reaction mechanism, owing to the simplicity of the eikonal scattering waves and the assumption of a single-step process.

A question of interest is the antisymmetrization of the full projectile-target scattering wave function. At intermediate energies $(\sim 100 \mathrm{MeV} /$ nucleon $)$, this effect is usually neglected. In the Glauber formalism of knockout reactions the scattering waves are calculated from an optical potential based on nucleon-nucleon scattering cross sections. A rough treatment of antisymmetrization is obtained by the manifestation of medium modification of the nucleon-nucleon cross section. Knowledge of the medium modification of the nucleonnucleon $(N N)$ cross section is necessary for an adequate numerical modeling of heavy-ion collision dynamics in central collisions (see, e.g., Ref. [12] and references therein). In these collisions, the ultimate purpose is to extract information about the nuclear equation of state by studying global collective variables describing the collision process. In direct reactions, such as knockout reactions, the medium effects on the $N N$ cross sections are much smaller because mostly low nuclear densities are probed. The goal in this work is to identify if medium modifications of $N N$ scattering modify appreciably the cross sections in knockout reactions. A systematic study of this effect in the literature is still lacking and is the focal point of this article.

Medium modifications of $N N$ cross sections are usually treated within the Brueckner-Hartree-Fock theory, where the $G$ matrix serves as the in-medium scattering amplitude, with medium effects being introduced through the self-consistent nuclear mean field and Pauli blocking. The literature in this subject is very long (see, e.g., Refs. [13-16]). In addition to being a fundamental input for nuclear reactions at high energies, the in-medium cross sections provide an immediate connection with the nucleon mean free path, $\lambda$, one of the most fundamental quantities characterizing the propagation of nucleons through matter. In turn, $\lambda$ enters the calculation of 
the nuclear transparency function where the latter is related to the total reaction cross section, $\sigma_{R}$, of a nucleus [17].

In this work we include medium effects of the $N N$ cross section in knockout reactions with a simple geometrical treatment of Pauli blocking and also with more elaborated Dirac-Brueckner results in terms of baryon densities. We focus specifically on symmetric nuclear matter. We calculate knockout cross sections and momentum distributions for selected reactions. After the introductory remarks in this section, in Sec. II we describe the formalism used in our calculations. Section III contains our numerical results. We conclude in Sec. IV with our summary.

\section{KNOCKOUT REACTIONS}

\section{A. Medium modification of nucleon-nucleon cross sections}

The free (total) $N N$ cross sections were taken from the Particle Data Group [18]. For our practical purposes, we have developed new fits for the free $N N$ cross sections, separated into three energy intervals, by means of the expressions

$$
\sigma_{p p}=\left\{\begin{array}{l}
19.6+4253 / E-375 / \sqrt{E}+3.86 \times 10^{-2} E \\
(\text { for } E<280 \mathrm{MeV}), \\
32.7-5.52 \times 10^{-2} E+3.53 \times 10^{-7} E^{3} \\
\quad-2.97 \times 10^{-10} E^{4} \\
(\text { for } 280 \mathrm{MeV} \leqslant E<840 \mathrm{MeV}), \\
50.9-3.8 \times 10^{-3} E+2.78 \times 10^{-7} E^{2} \\
\quad+1.92 \times 10^{-15} E^{4} \\
(\text { for } 840 \mathrm{MeV} \leqslant E \leqslant 5 \mathrm{GeV})
\end{array}\right.
$$

for proton-proton collisions and

$$
\sigma_{n p}=\left\{\begin{array}{l}
89.4-2025 / \sqrt{E}+19108 / E-43535 / E^{2} \\
(\text { for } E<300 \mathrm{MeV}), \\
14.2+5436 / E+3.72 \times 10^{-5} E^{2}-7.55 \times 10^{-9} E^{3} \\
(\text { for } 300 \mathrm{MeV} \leqslant E<700 \mathrm{MeV}), \\
33.9+6.1 \times 10^{-3} E-1.55 \times 10^{-6} E^{2} \\
\quad+1.3 \times 10^{-10} E^{3} \\
(\text { for } 700 \mathrm{MeV} \leqslant E \leqslant 5 \mathrm{GeV})
\end{array}\right.
$$

for proton-neutron collisions. $E$ is the projectile laboratory energy. The coefficients in the preceding equations have been obtained by a least squares fit to the $N N$ cross-section experimental data over a variety of energies, ranging from $10 \mathrm{MeV}$ to $5 \mathrm{GeV}$. In Fig. 1 these fits are represented by a solid line, whereas the filled circles are the experimental data from Ref. [18].

Most practical studies of medium corrections of $N N$ scattering are done by considering the effective two-nucleon interaction in infinite nuclear matter, or $G$ matrix, as a solution

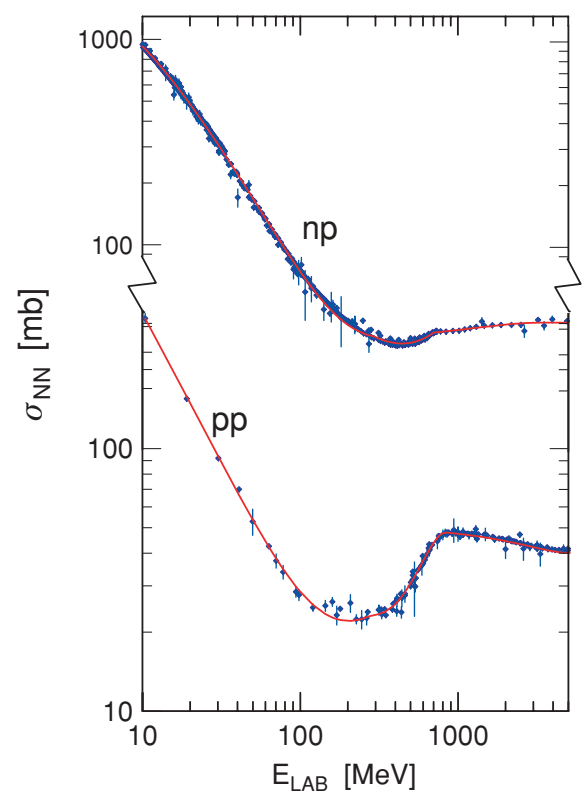

FIG. 1. (Color online) Least squares fit (solid curves) to the $N N$ cross section described by Eqs. (1) and (2). The experimental data are from Ref. [18].

of the Bethe-Goldstone equation [19],

$$
\begin{aligned}
\left\langle\mathbf{k}\left|\mathrm{G}\left(\mathbf{P}, \rho_{1}, \rho_{2}\right)\right| \mathbf{k}_{0}\right\rangle & \\
= & \left\langle\mathbf{k}\left|\mathrm{v}_{N N}\right| \mathbf{k}_{0}\right\rangle-\int \frac{d^{3} k^{\prime}}{(2 \pi)^{3}} \\
& \times \frac{\left\langle\mathbf{k}\left|\mathrm{v}_{N N}\right| \mathbf{k}^{\prime}\right\rangle Q\left(\mathbf{k}^{\prime}, \mathbf{P}, \rho_{1}, \rho_{2}\right)\left\langle\mathbf{k}^{\prime}\left|\mathrm{G}\left(\mathbf{P}, \rho_{1}, \rho_{2}\right)\right| \mathbf{k}_{0}\right\rangle}{E\left(\mathbf{P}, \mathbf{k}^{\prime}\right)-E_{0}-i \epsilon},
\end{aligned}
$$

with $\mathbf{k}_{0}, \mathbf{k}$, and $\mathbf{k}^{\prime}$ the initial, final, and intermediate relative momenta of the $N N$ pair, $\mathbf{k}=\left(\mathbf{k}_{1}-\mathbf{k}_{2}\right) / 2$ and $\mathbf{P}=\left(\mathbf{k}_{1}+\right.$ $\left.\mathbf{k}_{2}\right) / 2$. If energy and momentum are conserved in the binary collision, $\mathbf{P}$ is conserved in magnitude and direction, and the magnitude of $\mathbf{k}$ is also conserved. $\mathrm{v}_{N N}$ is the $N N$ potential. $E$ is the energy of the two-nucleon system, and $E_{0}$ is the same quantity on shell. Thus, $E(\mathbf{P}, \mathbf{k})=e(\mathbf{P}+\mathbf{k})+e(\mathbf{P}-\mathbf{k})$, with $e$ the single-particle energy in nuclear matter. It is also implicit in Eq. (3) that the final momenta $\mathbf{k}$ of the $N N$ pair also lie outside the range of occupied states.

Equation (3) is density dependent owing to the presence of the Pauli projection operator $Q$, defined by

$$
Q\left(\mathbf{k}, \mathbf{P}, \rho_{1}, \rho_{2}\right)= \begin{cases}1 & \text { if } k_{1,2}>k_{F 1, F 2}, \\ 0 & \text { otherwise }\end{cases}
$$

with $k_{1,2}$ the magnitude of the momenta of each nucleon. $Q$ prevents scattering into occupied intermediate states. The Fermi momenta $k_{F 1, F 2}$ are related to the proton and neutron densities by means of the zero-temperature density approximation, $k_{F i}=\left(3 \pi^{2} \rho_{i}\right)^{1 / 3}$. For finite nuclei, one usually replaces $\rho_{i}$ with the local densities to obtain the local Fermi momenta. This is obviously a rough approximation, but very practical and extensively used in the literature.

Only by means of several approximations, can Eq. (3) be related to $N N$ cross sections. If one neglects the medium modifications of the nucleon-mass and scattering through 
intermediate states, the medium modification of the $N N$ cross sections can be accounted for by the geometrical factor $Q$ only; that is,

$$
\sigma_{N N}\left(k, \rho_{1}, \rho_{2}\right)=\int \frac{d \sigma_{N N}^{\text {free }}}{d \Omega} Q\left(k, P, \rho_{1}, \rho_{2}\right) d \Omega,
$$

where $Q$ is now a simplified geometrical condition on the available scattering angles for the scattering of the $N N$ pair to unoccupied final states.

A usual approximation for the Pauli blocking is to assume that the effect of the $Q$ operator is equivalent to a restricted angular integration in the domain (for symmetric nuclear matter)

$$
\frac{k_{F}^{2}-P^{2}-k^{2}}{2 P k} \leqslant \cos \theta \leqslant \frac{P^{2}+k^{2}-k_{F}^{2}}{2 P k} .
$$

The integral in Eq. (5) becomes zero if the upper limit is negative [as determined by the condition in Eq. (4)], whereas the full integration range is used if the upper limit is greater than 1. [Notice that the average angle $\theta$ in Eq. (6), namely, the angle between the directions of $\mathbf{k}$ and $\mathbf{P}$, is also the colatitude of $\mathbf{k}$ in a coordinate system, where the $z$ axis is along $\mathbf{P}$ and, thus, in such a reference frame it coincides with the scattering angle to be integrated over in Eq. (5).] The method of using Eqs. (5) and (6) is not correct and misses an important part of the Pauli blocking geometry, as we show next.

A geometric description of the Pauli operator $Q$ was first studied by Clementel and Villi [20], who obtained an analytical expression for the scattering of a nucleon on a nucleon Fermi gas. By using the local-density approximation, their work has been widely used to describe Pauli-blocking in nucleon-nucleus scattering. Much later, in Ref. [21] (see also Appendix C of Ref. [17]), an expression was obtained for the geometrical $Q$ operator for nucleon scattering in asymmetric nuclear matter, involving two Fermi momentum spheres, one for the proton and another for the neutron. In contrast to Eq. (5), the expression obtained in Ref. [21] allows for $N N$ scattering with the relative momentum vector lying outside the symmetry axis of the two-Fermi-gas system.

As shown in Refs. [17,21], the Pauli blocking projection yields an average $N N$ cross section for two Fermi gases with relative momenta $\mathbf{k}_{0}$ (see Fig. 30 of Ref. [17]) given by

$$
\begin{aligned}
\sigma_{N N}\left(k, \rho_{1}, \rho_{2}\right)= & \int \frac{d^{3} k_{1} d^{3} k_{2}}{\left(4 \pi k_{F 1}^{3} / 3\right)\left(4 \pi k_{F 2}^{3} / 3\right)} \\
& \times \frac{2 q}{k_{0}} \sigma_{N N}^{\text {free }}(q) \frac{\Omega_{\text {Pauli }}}{4 \pi},
\end{aligned}
$$

where $2 \mathbf{q}=\mathbf{k}_{1}-\mathbf{k}_{2}-\mathbf{k}_{0}$.

Pauli blocking enters through the restriction that the magnitudes of the final nucleon momenta, $\left|\mathbf{k}_{1}^{\prime}\right|$ and $\left|\mathbf{k}_{2}^{\prime}\right|$, lie outside the Fermi spheres, with radii $k_{F 1}$ and $k_{F 2}$, respectively. This leads to a limited fraction of the solid angle into which the nucleons can scatter, $\Omega_{\text {Pauli }}$. It reads [21]

$$
\Omega_{\text {Pauli }}=4 \pi-2\left(\Omega_{a}+\Omega_{b}-\bar{\Omega}\right),
$$

where $\Omega_{a}$ and $\Omega_{b}$ specify the excluded solid angles for each nucleon, and $\bar{\Omega}$ represents the geometric intersection of the solid angles $\Omega_{a}$ and $\Omega_{b}$,

$$
\Omega_{a}=2 \pi\left(1-\cos \theta_{a}\right), \quad \Omega_{b}=2 \pi\left(1-\cos \theta_{b}\right),
$$

where

$$
\begin{aligned}
& \cos \theta_{a}=\left(p^{2}+q^{2}-k_{F 1}^{2}\right) / 2 p q, \\
& \cos \theta_{b}=\left(p^{2}+q^{2}-k_{F 2}^{2}\right) / 2 p q,
\end{aligned}
$$

with $2 \mathbf{p}=\mathbf{k}_{1}+\mathbf{k}_{2}+\mathbf{k}_{0}$.

For $\bar{\Omega}$ there are two possibilities:

(i) $\bar{\Omega}=\Omega_{i}\left(\theta, \theta_{a}, \theta_{b}\right)+\Omega_{i}\left(\pi-\theta, \theta_{a}, \theta_{b}\right)$, for $\theta+\theta_{a}+\theta_{b}>\pi$,

(ii) $\bar{\Omega}=\Omega_{i}\left(\theta, \theta_{a}, \theta_{b}\right), \quad$ for $\theta+\theta_{a}+\theta_{b} \leqslant \pi$,

where $\theta$ is given by

$$
\cos \theta=\left(k^{2}-p^{2}-b^{2}\right) / 2 p b,
$$

where $\mathbf{b}=\mathbf{k}-\mathbf{p}$.

The solid angle $\Omega_{i}$ has the following values

(i) $\Omega_{i}=0$ for $\theta \geqslant \theta_{a}+\theta_{b}$,

(ii)

$$
\begin{aligned}
\Omega_{i}= & 2\left[\cos ^{-1}\left(\gamma_{a b}\right)+\cos ^{-1}\left(\gamma_{b a}\right)\right. \\
& -\cos \theta_{a} \cos ^{-1}\left(\frac{\cos \theta_{b}-\cos \theta \cos \theta_{a}}{\sin \theta \sin \theta_{a}}\right) \\
& \left.-\cos \theta_{b} \cos ^{-1}\left(\frac{\cos \theta_{a}-\cos \theta \cos \theta_{b}}{\sin \theta \sin \theta_{b}}\right)\right]
\end{aligned}
$$$$
\text { for }\left|\theta_{b}-\theta_{a}\right| \leqslant \theta \leqslant \theta_{a}+\theta_{b},
$$

(iii) $\Omega_{i}=\Omega_{b}$ for $\theta_{b} \leqslant \theta_{a}, \quad \theta \leqslant\left|\theta_{b}-\theta_{a}\right|$,

(iv) $\Omega_{i}=\Omega_{a}$ for $\theta_{a} \leqslant \theta_{b}, \quad \theta \leqslant\left|\theta_{b}-\theta_{a}\right|$,

where

$\gamma_{j m}=\frac{\cos \theta_{m}-\cos \theta \cos \theta_{j}}{\sin \theta_{j}\left(\cos ^{2} \theta_{j}+\cos ^{2} \theta_{m}-2 \cos \theta \cos \theta_{j} \cos \theta_{m}\right)^{1 / 2}}$.

The integrals over $\mathbf{k}_{1}$ and $\mathbf{k}_{2}$ in Eq. (7) reduce to a fivefold integral owing to cylindrical symmetry. The remaining integrals have to be performed numerically. One sees that for two Fermi gases the problem is much more complicated than the one studied in Ref. [20]. For symmetric nuclear matter, that is, $k_{F} \equiv k_{F 1}=k_{F 2}$, the problem is still much more complicated than implied by Eq. (5), although many of the preceding terms simplify because in this case $\theta_{a}=\theta_{b}$ [22].

The numerical calculations can be simplified if we assume that the free $N N$ cross section entering Eq. (7) is isotropic. This is another rough approximation because the anisotropy of the free $N N$ cross section is markedly manifest at large energies $[13,14]$. In the isotropic case, we have devised a formula that fits the numerical integration in Eq. (7) to within $1 \%$. The parametrization reads

$$
\begin{aligned}
\sigma_{N N}\left(E, \rho_{1}, \rho_{2}\right)= & \sigma_{N N}^{\text {free }}(E) \frac{1}{1+1.892\left(\frac{\left|\rho_{1}-\rho_{2}\right|}{\tilde{\rho} \rho_{0}}\right)^{2.75}} \\
& \times \begin{cases}1-\frac{37.02 \tilde{\rho}^{2 / 3}}{E} & \text { if } E>46.27 \tilde{\rho}^{2 / 3}, \\
\frac{E}{231.38 \tilde{\rho}^{2 / 3}} & \text { if } E \leqslant 46.27 \tilde{\rho}^{2 / 3},\end{cases}
\end{aligned}
$$


where $E$ is the laboratory energy in $\mathrm{MeV}, \tilde{\rho}=\left(\rho_{1}+\rho_{2}\right) / \rho_{0}$, with $\rho_{0}=0.17 \mathrm{fm}^{-3}$.

The Brueckner method goes beyond a treatment of Pauli blocking, generating medium effects from $N N$ potentials such as the Bonn potential. An example is the work presented in Refs. [13,14], where a simple parametrization was given, which we from now on refer to as Brueckner theory. It reads (the misprinted factor 0.0256 in Ref. [14] has been corrected to 0.00256 )

$$
\sigma_{n p}=\left[31.5+0.092\left|20.2-E^{0.53}\right|^{2.9}\right] \frac{1+0.0034 E^{1.51} \rho^{2}}{1+21.55 \rho^{1.34}},
$$

$\sigma_{p p}=\left[23.5+0.00256\left(18.2-E^{0.5}\right)^{4.0}\right] \frac{1+0.1667 E^{1.05} \rho^{3}}{1+9.704 \rho^{1.2}}$.

A modification of the preceding parametrization was done in Ref. [23], which consisted in combining the free $N N$ cross sections parametrized in Ref. [24] with the Brueckner theory results of Refs. $[13,14]$. Their parametrization, which tends to reproduce better the nucleus-nucleus reaction cross sections, is

$$
\begin{aligned}
\sigma_{n p}= & {\left[-70.67-18.18 \beta^{-1}+25.26 \beta^{-2}+113.85 \beta\right] } \\
& \times \frac{1+20.88 E^{0.04} \rho^{2.02}}{1+35.86 \rho^{1.9}}, \\
\sigma_{p p}= & {\left[13.73-15.04 \beta^{-1}+8.76 \beta^{-2}+68.67 \beta^{4}\right] } \\
& \times \frac{1+7.772 E^{0.06} \rho^{1.48}}{1+18.01 \rho^{1.46}},
\end{aligned}
$$

where $\beta=\sqrt{1-1 / \gamma^{2}}$ and $\gamma=E[\mathrm{MeV}] / 931.5+1$. We denote Eq. (16) as the phenomenological parametrization.

In Figs. 2-5 we compare the several parametrizations previously described and we postpone the discussion of their details to Sec. III.

\section{B. Nucleon knockout reactions}

The momentum distributions of the projectilelike residues in one-nucleon knockout are a measure of the spatial extent of the wave function of the struck nucleon, while the cross section for the nucleon removal scales with the occupation amplitude, or probability (spectroscopic factor), for the given single-particle configuration in the projectile ground state. The longitudinal momentum distributions are given by (see, e.g., Refs. [6,25,26])

$$
\begin{aligned}
\frac{d \sigma_{\text {str }}}{d k_{z}}= & \left(C^{2} S\right) \frac{1}{2 \pi} \frac{1}{2 l+1} \sum_{m} \int_{0}^{\infty} d^{2} b_{n}\left[1-\left|S_{n}\left(b_{n}\right)\right|^{2}\right] \\
& \times \int_{0}^{\infty} d^{2} \rho\left|S_{c}\left(b_{c}\right)\right|^{2}\left|\int_{-\infty}^{\infty} d z \exp \left[-i k_{z} z\right] \psi_{l m}(\mathbf{r})\right|^{2},
\end{aligned}
$$

where $k_{z}$ represents the longitudinal component of $\mathbf{k}_{c}$ (final momentum of the core of the projectile nucleus), $\left(C^{2} S\right)$ is the spectroscopic factor, and $\psi_{l m}(\mathbf{r})$ is the wave function of the core plus (valence) nucleon system $(c+n)$ in a state with single-particle angular momentum $l, m$. In this equation,

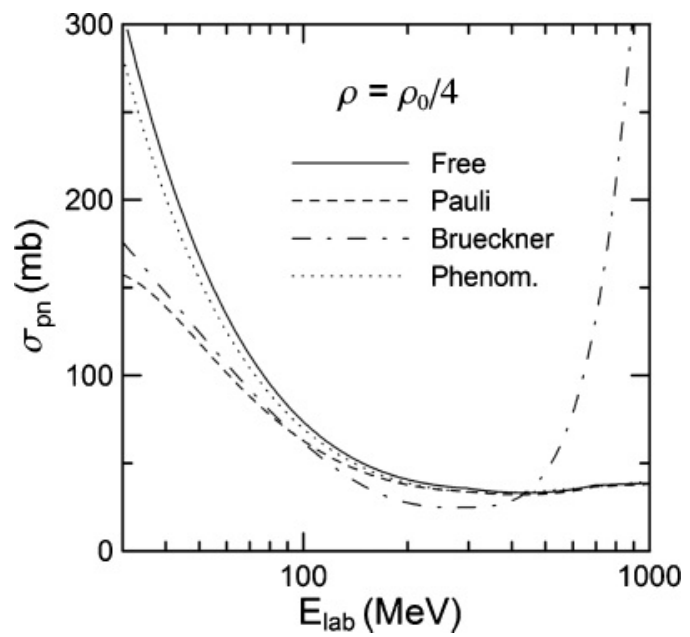

FIG. 2. Parametrizations of proton-neutron cross sections as a function of the laboratory energy. The solid line is the parametrization of the free $\sigma_{p n}$ cross section given by Eq. (2). The other curves include medium effects for symmetric nuclear matter for $\rho=\rho_{0} / 4$, where $\rho_{0}=0.17 \mathrm{fm}^{-3}$. The dashed curve includes the geometrical effects of Pauli blocking, as described by Eq. (14). The dashed-dotted curve is the result of the Brueckner theory [Eq. (15)] and the dotted curve is the phenomenological parametrization [Eq. (16)].

$$
\begin{aligned}
\mathbf{r} \equiv(\rho, z, \phi) & =\mathbf{r}_{n}-\mathbf{r}_{c}, \text { so that } \\
b_{c} & =\left|\rho-\mathbf{b}_{n}\right|=\sqrt{\rho^{2}+b_{n}^{2}-2 \rho b_{n} \cos \phi} \\
& =\sqrt{r^{2} \sin ^{2} \theta+b_{n}^{2}-2 r \sin \theta b_{n} \cos \phi}
\end{aligned}
$$

$S_{i}(b)$ are the $S$ matrices for core-target and nucleon-target scattering obtained from the nuclear ground-state densities and the $N N$ cross sections by the relation [27] $S(b)=\exp [i \phi(b)]$, with

$$
\phi_{N}(b)=i \frac{\sigma_{N N}}{4 \pi} \int_{0}^{\infty} d q q \rho_{p}(q) \rho_{t}(q) J_{0}(q b),
$$

where $\rho_{p, t}(q)$ is the Fourier transform of the nuclear density of the projectile (nucleon or core) and the target nucleus, and $\sigma_{N N}$

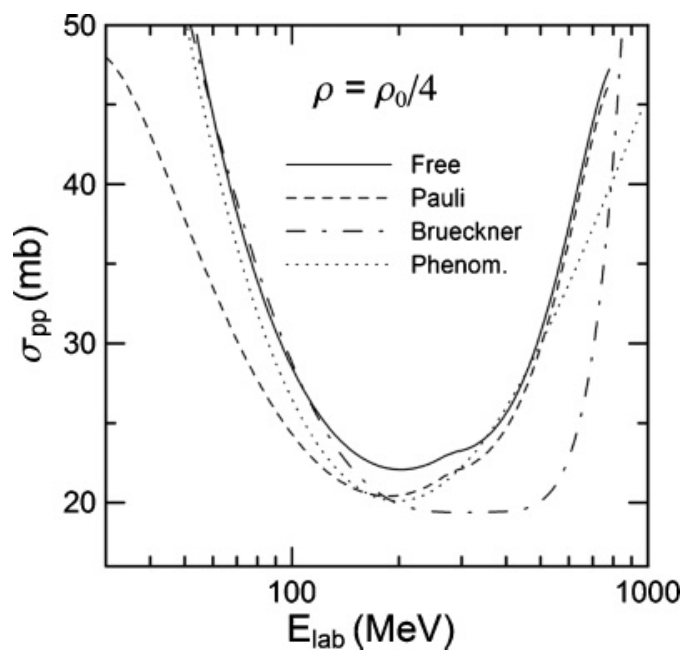

FIG. 3. Same as in Fig. 2, but for $p p$ collisions. 


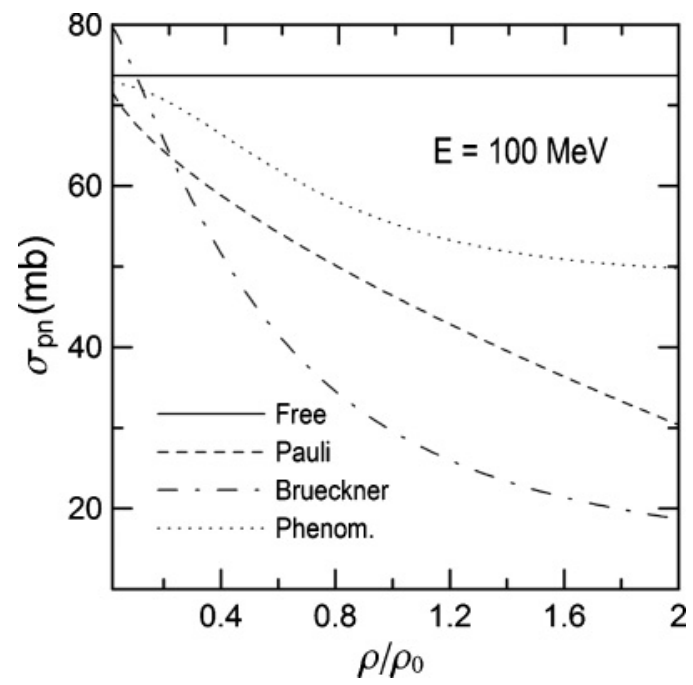

FIG. 4. Parametrizations of proton-neutron cross sections as a function of the nuclear-matter density (in units of $\rho_{0}=0.17 \mathrm{fm}^{-3}$ ). The solid line is the parametrization of the free $\sigma_{p n}$ cross section given by Eq. (2). The other curves include medium effects for symmetric nuclear matter for laboratory energy $E=100 \mathrm{MeV}$. The dashed curve includes the geometrical effects of Pauli blocking, as described by Eq. (14). The dashed-dotted curve is the result of the Brueckner theory [Eq. (15)], and the dotted curve is the phenomenological parametrization [Eq. (16)].

is the $N N$ total cross section. One needs to add the Coulomb phase to the nuclear eikonal phase of Eq. (19). This is done by using a sharp-cutoff expression for the Coulomb phase, as explained in Refs. $[6,26]$.

The first term inside the integrals in Eq. (17), $1-\left|S_{n}\right|^{2}$, represents the probability for the knockout of the nucleon from its location at $b_{n}$, whereas the second integral carries the term $\left|S_{c}\right|^{2}$, which is the probability of core survival at impact parameter $b_{c}$. These results arise naturally by using eikonal scattering waves [27]. For the transverse momentum

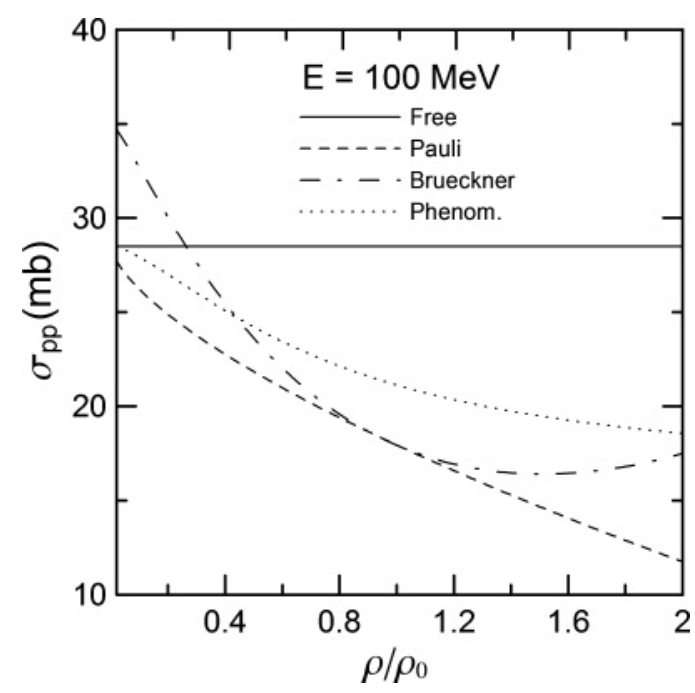

FIG. 5. Same as in Fig. 4, but for proton-proton collisions. distributions, the same formalism yields

$$
\begin{aligned}
\frac{d \sigma_{\text {str }}}{d^{2} k_{c}^{\perp}}= & \left(C^{2} S\right) \frac{1}{(2 \pi)^{2}} \frac{1}{2 l+1} \int_{0}^{\infty} d^{2} b_{n}\left[1-\left|S_{n}\left(b_{n}\right)\right|^{2}\right] \\
& \times \sum_{m} \int_{-\infty}^{\infty} d z\left|\int d^{2} \rho \exp \left(-i \mathbf{k}_{c}^{\perp} \cdot \rho\right) S_{c}\left(b_{c}\right) \psi_{l m}(\mathbf{r})\right|^{2},
\end{aligned}
$$

where $\mathbf{k}_{c}^{\perp}$ is the perpendicular component of $\mathbf{k}_{c}$.

The total stripping cross section can be obtained by integrating either Eq. (17) or Eq. (20). One obtains

$$
\begin{aligned}
\sigma_{\text {str }}= & \left(C^{2} S\right) \frac{2 \pi}{2 l+1} \sum_{m} \int_{0}^{\infty} d b_{n} b_{n}\left[1-\left|S_{n}\left(b_{n}\right)\right|^{2}\right] \\
& \times \int d^{3} r\left|S_{c}\left(b_{c}\right)\right|^{2}\left|\psi_{l m}(\mathbf{r})\right|^{2} .
\end{aligned}
$$

The total diffraction dissociation cross section is given by [26]

$$
\begin{aligned}
\sigma_{\mathrm{dif}}= & \left(C^{2} S\right) \frac{2 \pi}{2 l+1} \sum_{m} \int_{0}^{\infty} d b_{n} b_{n} \\
& \times\left\{\int d^{3} r\left|S_{n}\left(b_{n}\right) S_{c}\left(b_{c}\right) \psi_{l m}(\mathbf{r})\right|^{2}\right. \\
& \left.-\sum_{m^{\prime}}\left|\int d^{3} r \psi_{l m^{\prime}}(\mathbf{r}) S_{c}\left(b_{c}\right) S_{n}\left(b_{n}\right) \psi_{l m}(\mathbf{r})\right|^{2}\right\} .
\end{aligned}
$$

To render the calculations practical, for a nucleus-nucleus collision with a given impact parameter $b$, we have obtained an effective local density for protons and neutrons by taking the point along the impact parameter direction where the two densities (one from the projectile and the other from the target) cross each other. This effective density was then used in Eqs. (14)-(16)

In the following we use a modified version of the code MOMDIS [26], which includes the new aspects of momentum distributions discussed in this article. Because we want to make a theoretical study of the medium effects of the $N N$ cross sections, we do not compare directly to experiments and we use spectroscopic factors $\left(C^{2} S\right)$ equal to the unity. To generate the wave functions and $S$-matrices, we use the same parameters as in Refs. [6] and [26].

\section{RESULTS AND DISCUSSION}

There are marked differences between the parametrization of the Brueckner (15), the geometrical Pauli blocking (14) and the phenomenological one (16). An example is given in figure 2 where the several parametrizations of proton-neutron cross sections are shown as a function of the laboratory energy. The solid line is the parametrization of the free $\sigma_{p n}$ cross section given by Eq. (2). The other curves include medium effects for symmetric nuclear matter for $\rho=\rho_{0} / 4$, where $\rho_{0}=0.17 \mathrm{fm}^{-3}$. The dashed curve includes the geometrical effects of Pauli blocking, as described by Eq. (14). The dasheddotted curve is the result of the Brueckner theory [Eq. (15)] and the dotted curve is the phenomenological parametrization, Eq. (16). The large deviation of the parametrization of the 
Brueckner results at large energies is not physical because Eq. (15) is only a good parametrization of the Brueckner theory in the energy range of 50-300 [13,14]. At energies above $300 \mathrm{MeV}$ inelastic channels have to be incorporated. However, the other differences are real, especially those at lower energies. Pauli blocking effectively reduces the in-medium $n p$ cross section. This is not so apparent in the phenomenological parametrization.

The preceding observations cannot be extended to the $p p$ cross sections, which are shown in Fig. 3. Here we see that the Pauli-blocking correction decreases the cross section much more than in the other cases. Some important differences are also clearly visible at larger energies, $E \gtrsim 100 \mathrm{MeV} /$ nucleon.

Figure 4 shows the proton-neutron cross sections as a function of the nuclear matter density (in units of $\rho_{0}=0.17 \mathrm{fm}^{-3}$ ), for a proton laboratory energy of $E_{\text {lab }}=100 \mathrm{MeV}$. The solid line is the parametrization of the free $\sigma_{p n}$ cross section given by Eq. (2). The other curves include medium effects for symmetric nuclear matter. The dashed curve includes the geometrical effects of Pauli blocking, as described by Eq. (14). The dashed-dotted curve is the result of the Brueckner theory [Eq. (15)] and the dotted curve is the phenomenological parametrization [Eq. (16)]. Figure 5 shows the same as in Fig. 4, but for proton-proton collisions. One notices that the $N N$ cross sections differ appreciably at large densities but they become close to the free cross sections at low densities.

To test the influence of the medium effects in nucleon knockout reactions, we consider the removal of the $l=0$ halo neutron of ${ }^{15} \mathrm{C}$, bound by $1.218 \mathrm{MeV}$, and the $l=0$ neutron knockout from ${ }^{34} \mathrm{Ar}$, bound by $17.06 \mathrm{MeV}$. The reactions studied here are the ${ }^{9} \mathrm{Be}\left({ }^{15} \mathrm{C},{ }^{14} \mathrm{C}_{g s}\right)$ and ${ }^{9} \mathrm{Be}\left({ }^{34} \mathrm{Ar},{ }^{33} \mathrm{Ar}\left(1 / 2^{+}\right)\right)$. The total cross sections as a function of the bombarding energy are shown in Figs. 6 and 7. The solid curve is obtained with the use of free nucleon-nucleon cross sections. The dashed

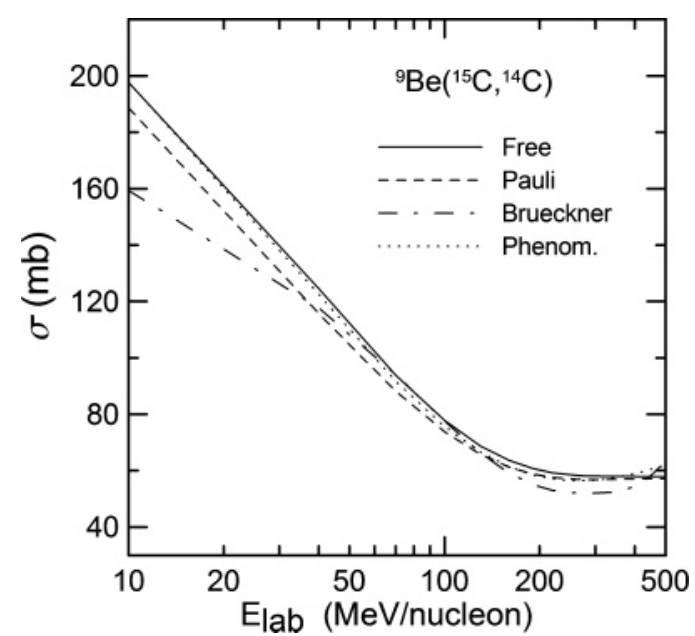

FIG. 6. Total knockout cross sections for removing the $l=0$ halo neutron of ${ }^{15} \mathrm{C}$, bound by $1.218 \mathrm{MeV}$, in the reaction ${ }^{9} \mathrm{Be}\left({ }^{15} \mathrm{C},{ }^{14} \mathrm{C}_{g s}\right)$. The solid curve is obtained with the use of free $N N$ cross sections. The dashed curve includes the geometrical effects of Pauli blocking, as described by Eq. (14). The dashed-dotted curve is the result using the Brueckner theory [Eq. (15)] and the dotted curve is the phenomenological parametrization [Eq. (16)].

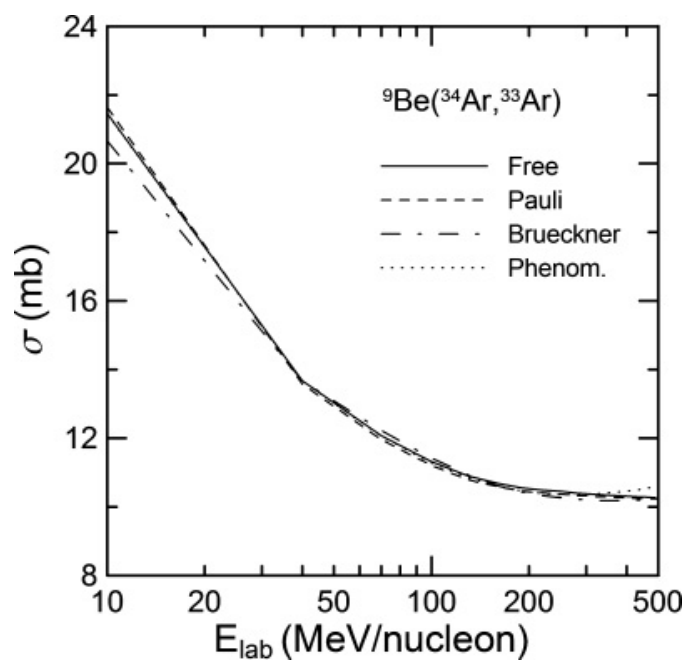

FIG. 7. Same as in Fig. 6, but for the removal of the $l=0$ neutron bound by $17.06 \mathrm{MeV}$ in the reaction ${ }^{9} \mathrm{Be}\left({ }^{34} \mathrm{Ar},{ }^{33} \mathrm{Ar}\left(1 / 2^{+}\right)\right)$.

curve includes the geometrical effects of Pauli blocking, as described by Eq. (14). The dashed-dotted curve is the result using the Brueckner theory [Eq. (15)] and the dotted curve is the phenomenological parametrization [Eq. (16)].

The medium effects owing to different treatments are more visible for the ${ }^{9} \mathrm{Be}\left({ }^{15} \mathrm{C},{ }^{14} \mathrm{C}_{g s}\right)$ reaction. For ${ }^{9} \mathrm{Be}\left({ }^{34} \mathrm{Ar},{ }^{33} \mathrm{Ar}\left(1 / 2^{+}\right)\right)$the differences are almost not visible, as shown in Fig. 7. The same happens for the $l=2$ neutron removal reaction leading to a final $3 / 2^{+}$level bound by 18.42 MeV. A similar behavior as for the ${ }^{9} \mathrm{Be}\left({ }^{15} \mathrm{C},{ }^{14} \mathrm{C}_{g s}\right)$ reaction is found for the removal of the halo neutron in the nucleon knockout ${ }^{9} \mathrm{Be}\left({ }^{11} \mathrm{Be},{ }^{10} \mathrm{Be}\right)$ bound by $0.504 \mathrm{MeV}$. It is thus apparent that the corrections owing to the medium effects are more evident for the knockout out from loosely bound states. Knockout reactions are also more sensitive to in-medium corrections of the $N N$ cross sections than the total reaction cross sections, as first pointed out in Ref. [17].

In Table I we show our results for the stripping, diffraction dissociation, and total nucleon cross section (in $\mathrm{mb}$ ) for the knockout reactions ${ }^{9} \mathrm{Be}\left({ }^{11} \mathrm{Be},{ }^{10} \mathrm{Be}\right),{ }^{9} \mathrm{Be}\left({ }^{15} \mathrm{C},{ }^{14} \mathrm{C}\right)$, and ${ }^{9} \mathrm{Be}\left({ }^{34} \mathrm{Ar},{ }^{33} \mathrm{Ar}\right)$ at $40 \mathrm{MeV} /$ nucleon. For the reaction ${ }^{9} \mathrm{Be}\left({ }^{34} \mathrm{Ar},{ }^{33} \operatorname{Ar}\left(3 / 2^{+}\right)\right)$the values are $\sigma_{\text {dis }}=2.36 \mathrm{mb}, \sigma_{\text {str }}=$ $9.16 \mathrm{mb}$, and $\sigma_{\text {tot }}=11.5 \mathrm{mb}$ and have the same value,

TABLE I. Cross sections in $\mathrm{mb}$ at $40 \mathrm{MeV} /$ nucleon for nucleon knockout of a few selected reactions.

\begin{tabular}{|c|c|c|c|c|c|}
\hline Reaction & $\sigma$ & Free & Pauli & Brueckner & Pheno. \\
\hline \multirow[t]{3}{*}{${ }^{9} \mathrm{Be}\left({ }^{11} \mathrm{Be},{ }^{10} \mathrm{Be}\right)$} & $\sigma_{\mathrm{dif}}$ & 47.6 & 36.9 & 45.7 & 45.2 \\
\hline & $\sigma_{\text {str }}$ & 151 & 144 & 139 & 149 \\
\hline & $\sigma_{\text {tot }}$ & 198 & 181 & 185 & 194 \\
\hline \multirow[t]{3}{*}{${ }^{9} \mathrm{Be}\left({ }^{15} \mathrm{C},{ }^{14} \mathrm{C}\right)$} & $\sigma_{\text {dif }}$ & 25.3 & 19.9 & 21.3 & 24.0 \\
\hline & $\sigma_{\text {str }}$ & 99.8 & 95.8 & 96.5 & 98.5 \\
\hline & $\sigma_{\text {tot }}$ & 125 & 116 & 118 & 123 \\
\hline \multirow[t]{3}{*}{${ }^{9} \operatorname{Be}\left({ }^{34} \mathrm{Ar},{ }^{33} \operatorname{Ar}\left(1 / 2^{+}\right)\right)$} & $\sigma_{\text {dif }}$ & 2.69 & 2.63 & 2.66 & 2.68 \\
\hline & $\sigma_{\text {str }}$ & 11.0 & 10.9 & 11.0 & 11.0 \\
\hline & $\sigma_{\text {tot }}$ & 13.6 & 13.5 & 13.6 & 13.6 \\
\hline
\end{tabular}


TABLE II. Cross sections in $\mathrm{mb}$ at $250 \mathrm{MeV} /$ nucleon for nucleon knockout of a few selected reactions.

\begin{tabular}{lccccc}
\hline \hline Reaction & $\sigma$ & Free & Pauli & Brueckner & Pheno. \\
\hline${ }^{9} \mathrm{Be}\left({ }^{11} \mathrm{Be},{ }^{10} \mathrm{Be}\right)$ & $\sigma_{\text {dif }}$ & 11.0 & 10.3 & 8.64 & 10.0 \\
& $\sigma_{\text {str }}$ & 74.4 & 73.0 & 66.0 & 71.7 \\
${ }^{9} \mathrm{Be}\left({ }^{15} \mathrm{C},{ }^{14} \mathrm{C}\right)$ & $\sigma_{\text {tot }}$ & 85.8 & 83.1 & 75.0 & 81.7 \\
& $\sigma_{\text {dif }}$ & 5.14 & 4.78 & 3.90 & 4.63 \\
& $\sigma_{\text {str }}$ & 53.4 & 52.3 & 48.2 & 51.8 \\
${ }^{9} \mathrm{Be}\left({ }^{34} \mathrm{Ar},{ }^{33} \mathrm{Ar}\left(1 / 2^{+}\right)\right)$ & $\sigma_{\text {tot }}$ & 58.5 & 57.1 & 52.1 & 56.4 \\
& $\sigma_{\text {dif }}$ & 0.801 & 0.785 & 0.749 & 0.778 \\
& $\sigma_{\text {str }}$ & 9.62 & 9.55 & 9.47 & 9.55 \\
& $\sigma_{\text {tot }}$ & 10.5 & 10.4 & 10.2 & 10.4 \\
\hline \hline
\end{tabular}

within $1 \%$, for calculations using all of the $N N$ cross-section parametrizations, either Eq. (1) or (2) or Eqs. (14), (15), or (16).

In Table II we show the same as in Table I but for $E_{\text {lab }}=$ $250 \mathrm{MeV} /$ nucleon. For the reaction ${ }^{9} \mathrm{Be}\left({ }^{34} \mathrm{Ar},{ }^{33} \mathrm{Ar}\left(3 / 2^{+}\right)\right)$the values are $\sigma_{\text {dis }}=0.691 \mathrm{mb}, \sigma_{\text {str }}=8.62 \mathrm{mb}$, and $\sigma_{\text {tot }}=9.32 \mathrm{mb}$ and have the same value, and as in Table I, these values differ by less than $1 \%$, for all $N N$ cross-section parametrizations used in the calculations.

In Fig. 8 we plot the longitudinal momentum distributions for the reaction ${ }^{9} \mathrm{Be}\left({ }^{11} \mathrm{Be},{ }^{10} \mathrm{Be}\right)$ at $250 \mathrm{MeV} /$ nucleon. The calculations are done using Eq. (17). The diffraction dissociation cross sections have been calculated using the same profile of the momentum distribution owing to stripping, but with the total cross section normalized to Eq. (22). The different contributions (stripping and diffraction dissociation) to this reaction are given in Table II. In Fig. 8 the dashed curve is the cross section calculated using the $N N$ cross section from the Brueckner theory [Eq. (15)] and the solid curve is obtained the free cross section [Eq. (16)]. One sees that the momentum distributions are reduced by $10 \%$, about the same as the total cross sections, but the shape remains basically unaltered. If one

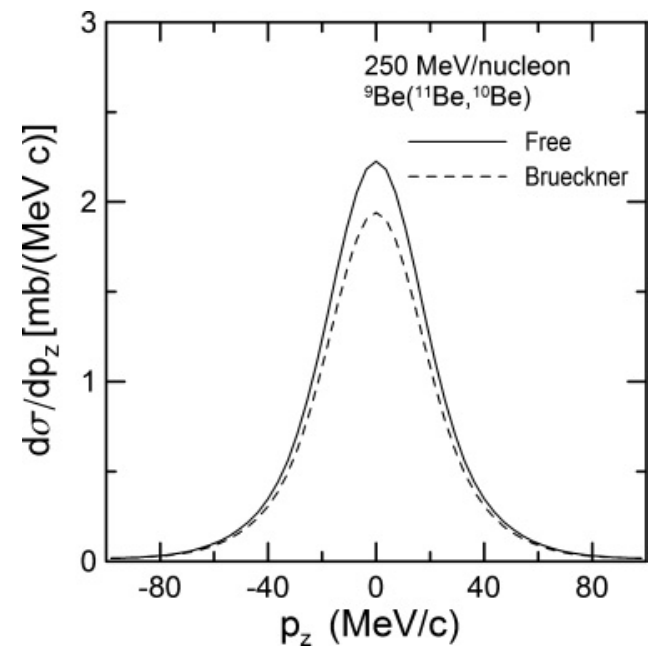

FIG. 8. Longitudinal momentum distribution for the residue in the ${ }^{9} \mathrm{Be}\left({ }^{11} \mathrm{Be},{ }^{10} \mathrm{Be}\right)$ reaction at $250 \mathrm{MeV} /$ nucleon. The dashed curve is the cross section calculated using the $N N$ cross section from the Brueckner theory [Eq. (15)] and the solid curve is obtained the free cross section [Eq. (16)].

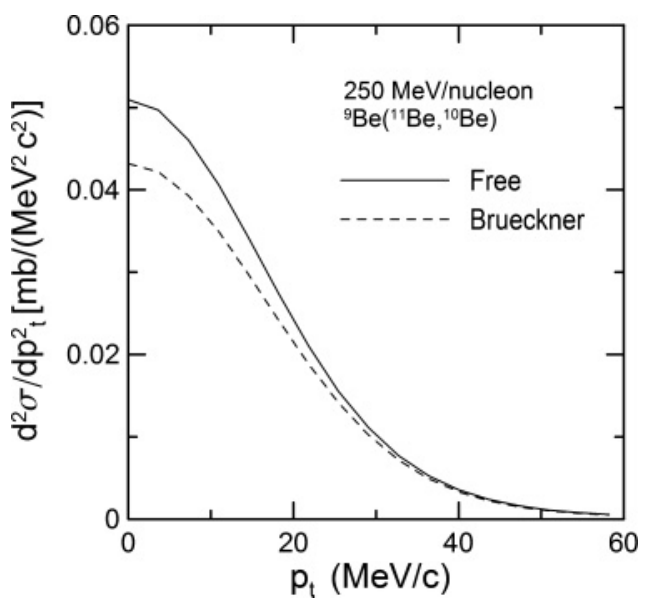

FIG. 9. Same as in Fig. 8, but for the transverse momentum distribution.

rescales the dashed curve to match the solid one, the differences in the width are not visible. We do not show the momentum distributions using the other two (Pauli and phenomenological) $N N$ cross sections because their shapes are the same as for the Brueckner case and only the area below the curve (total knockout cross section) changes.

In Fig. 9 we plot the transverse momentum distributions for the reaction ${ }^{9} \mathrm{Be}\left({ }^{11} \mathrm{Be},{ }^{10} \mathrm{Be}\right)$ at $250 \mathrm{MeV} /$ nucleon. The calculations are done using Eq. (20). As with Fig. 8, the dashed curve in Fig. 9 is the cross section calculated using the $N N$ cross section from the Brueckner theory [Eq. (15)] and the solid curve is obtained using the free cross section [Eq. (16)]. The changes on the profile of the momentum distribution are again visible, which is again ascribed to the difference of about $10 \%$ between the total cross sections. The forms of the momentum distributions are the same if the two curves are scaled to have the same area.

These results clearly show that the effects of $N N$ scattering in the medium on knockout reactions are worth considering, especially for reactions involving loosely bound halo nuclei. It is not clear, however, which of the several parametrizations of medium effects is more adequate for the precision required by experiments.

\section{SUMMARY}

The present work has extended the theory of one-nucleon stripping and diffraction dissociation reactions to cover the dependence of the nucleon knockout cross sections and momentum distributions on the medium modifications of the $N N$ cross sections. We included the most commonly used parametrizations in the literature and compared the effects of Pauli blocking from a simple geometrical picture to a more elaborated Dirac-Brueckner calculation, as well as phenomenological parametrizations. We have shown that the density dependencies vary rather strongly from model to model for reasons that are not yet clear.

We have also shown that the nucleon knockout reactions involving halo nuclei are more sensitive to medium modifications of the $N N$ cross section than in the case of the removal 
of more bound nucleons. The changes amount to $10 \%$ in some cases, especially at lower energies. However, owing to the average of the $N N$ cross sections over the local densities, the changes are not always predictable at higher energies. The stripping and diffraction dissociation cross sections decrease and increase in the same way whenever the $N N$ cross sections decrease or increase from one parametrization to another.

The momentum distributions are not appreciably different, except for their absolute normalization, when the $N N$ cross sections change with medium modifications. This has been verified for both longitudinal and transverse momentum distributions.

The simple study of the density-dependent $N N$ cross section adopted in this work shows that the calculations are sometimes sensitive to the value of the density-dependence method under consideration. Besides Pauli-blocking and medium changes in the $N N$ cross section, Fermi motion should probably play an important role in the nucleon knockout reactions and is worth further investigation.

No attempt has been done to compare to experimental results, which would probably affect the extracted values of the spectroscopic strengths in reactions with rare isotopes. This certainly deserves further theoretical study.

It is also worth mentioning that the magnitude of the corrections observed in this work, of about $10 \%$ for the total cross sections, are based on the optical limit (OL) of the Glauber multiple scattering theory. The OL means that only single binary $N N$ collisions are included. In the present work correlations within the projectile and target wave functions have been neglected. These have been studied, for example, in Ref. [28] or more recently in Ref. [7]. In these references, the influence of these correlations on the calculations has been studied and found to be also of the order of $10 \%$. This is of the same order of magnitude as the corrections observed in the present work. It is not clear if these two unrelated corrections will add up to a larger correction of the knockout cross sections, which could in fact modify appreciably the spectroscopic factors published in the literature where such corrections were not included. This also qualifies for further investigation.

It is worth mentioning that medium modifications of $N N$ scattering have also been studied in several publications related to $(p, 2 p)$ reactions (see, e.g., Refs. [29-31]). The medium effects were shown to play an important role on the total cross sections and on the spin observables.

\section{ACKNOWLEDGMENTS}

C.B. thanks Lie-Wen Chen for useful conversations. C.B. acknowledges support by the US Department of Energy under Grant Nos. DE-FG02-08ER41533 and DE-FC02-07ER41457 (UNEDF, SciDAC-2) and the Research Corporation. C.D.C. acknowledges the Brazilian agency FAPESP (Fundação de Amparo a Pesquisa do Estado de São Paulo) for financial support and the hospitality of the Department of Physics and Astronomy of Texas A\&M University-Commerce, where this work was done.
[1] C. A. Bertulani and K. W. McVoy, Phys. Rev. C 46, 2638 (1992)

[2] P. G. Hansen, Nature (London) 334, 194 (1998).

[3] J. A. Tostevin, J. Phys. G 25, 735 (1999).

[4] P. G. Hansen and J. A. Tostevin, Annu. Rev. Nucl. Part. Sci. 53, 219 (2003).

[5] A. Gade et al., Phys. Rev. C 77, 044306 (2008).

[6] C. A. Bertulani and P. G. Hansen, Phys. Rev. C 70, 034609 (2004).

[7] B. Abu-Ibrahim and Y. Suzuki, Phys. Rev. C 61, 051601(R) (2000).

[8] B. Abu-Ibrahim, Y. Ogawa, Y. Suzuki, and I. Tanihata, Comput. Phys. Commun. 151, 369 (2003).

[9] J. A. Tostevin and B. A. Brown, Phys. Rev. C 74, 064604 (2006).

[10] E. C. Simpson, J. A. Tostevin, D. Bazin, B. A. Brown, and A. Gade, Phys. Rev. Lett. 102, 132502 (2009).

[11] E. C. Simpson, J. A. Tostevin, D. Bazin, and A. Gade, Phys. Rev. C 79, 064621 (2009).

[12] B.-A. Li, L.-W. Chen, and C. M. Ko, Phys. Rep. 464, 113 (2008).

[13] G. Q. Li and R. Machleidt, Phys. Rev. C 48, 1702 (1993).

[14] G. Q. Li and R. Machleidt, Phys. Rev. C 49, 566 (1994).

[15] F. Sammarruca and P. Krastev, Phys. Rev. C 73, 014001 (2006).

[16] F. Sammarruca, Phys. Rev. C 77, 047301 (2008).
[17] M. Hussein, R. Rego, and C. A. Bertulani, Phys. Rep. 201, 279 (1991).

[18] W.-M. Yao et al., J. Phys. G 33, 1 (2006).

[19] L. C. Gomes, J. D. Walecka, and V. F. Weisskopf, Ann. Phys. (NY) 3, 241 (1958).

[20] E. Clementel and C. Villi, Nuovo Cimento 1, 176 (1955).

[21] C. A. Bertulani, Braz. J. Phys. 16, 380 (1986).

[22] C. A. Bertulani, J. Phys. G 27, L67 (2001).

[23] C. Xiangzhou, F. Jun, S. Wenqing, M. Yugang, W. Jiansong, and Y. Wei, Phys. Rev. C 58, 572 (1998).

[24] S. K. Charagi and S. K. Gupta, Phys. Rev. C 41, 1610 (1990).

[25] K. Hencken, G. Bertsch, and H. Esbensen, Phys. Rev. C 54, 3043 (1996).

[26] C. A. Bertulani and A. Gade, Comput. Phys. Commun. 175, 372 (2006).

[27] C. A. Bertulani and P. Danielewicz, Introduction to Nuclear Reactions (Taylor \& Francis, Inc., UK, 2004), Chap. 8.

[28] V. Franco and G. K. Varma, Phys. Rev. C 15, 1375 (1977).

[29] G. Jacob and Th. A. J. Maris, Rev. Mod. Phys. 38, 121 (1973).

[30] C. Samanta, N. S. Chant, P. G. Roos, A. Nadasen, J. Wesick, and A. A. Cowley, Phys. Rev. C 34, 1610 (1986).

[31] G. Krein, Th. A. J. Maris, B. B. Rodrigues, and E. A. Veit, Phys. Rev. C 51, 2646 (1995). 